\section{Microcurrent Point Stimulation Applied to Acupuncture Points for the Treatment of Non-Spe- cific Lower Back Pain}

\author{
Antoine Chevalier $^{1 *}$, Kelly Armstrong ${ }^{1}$ and Raman Gokal ${ }^{2}$ \\ ${ }^{1}$ University of Natural Health, IN and NIH, Bethesda, MD, USA \\ ${ }^{2}$ Department of Medicine, Royal Infirmary, University of Manchester, \\ Manchester, $U$
}

\begin{abstract}
Although acupuncture and microcurrent are widely used for chronic pain, there remains considerable controversy as to their therapeutic value. We aimed to determine in an observational study the effect size of DC Microcurrent Point Stimulation (MPS) applied to acupuncture points for 68 non-specific chronic back pain patients in a cohort analysis of treatment outcomes pre-post and follow-up MPS.

A detailed comparative analysis of the effects of DC MPS on patient pain levels when applied to standardized acupuncture pain protocol was evaluated; this entailed a standard protocol baseline VAS (VAS) pain scale before and after post electro-therapy and after a two day follow-up. A total of 68 patients with non-specific lower back pain were recruited, all of whom received MPS in 1 session. Pain intensity was measured by visual analog scale at baseline, post and 48 hour follow-up. The VAS response of this patient sample with chronic pain following MPS applied to standard acupuncture pain protocol reflected a statistically significant reduction of 3.088 (95\% $\mathrm{Cl}(2.715,3.462) ; \mathrm{P}=0.001)$. This corresponds to a mean reduction of $62 \%$; from 5.00 to 1.88 , in pain levels post treatment when compared to initial pain levels. There was also further statistically significant reduction of $0.632(33 \%)$ in follow-up pain scores, when compared to post pain levels [ $95 \% \mathrm{Cl}(0.295,0.969) ; p=0.0001]$, for a total end pain relief of $75 \%$. The positive results in this study could have significant and positive implications for patients afflicted with chronic back pain.
\end{abstract}

Keywords: Acupuncture; Back pain; Microcurrent Point Stimulation (MPS); Standard protocol

\section{Introduction}

Lower back pain is the single leading cause of disability worldwide, according to the global burden of disease 2010, with over one-half of all working Americans admit to having back pain symptoms each year

*Corresponding author: Antoine Chevalier, University of Natural Health, IN and NIH, Bethesda, MD, USA; 21010 South Bank st, \#5050, Sterling, VA 20165 USA, Tel: +1 3017688384; E-mail: antoine@healedandhappy.net

Citation: Chevalier A, Armstrong K, Gokal R (2016) Microcurrent Point Stimulation Applied to Acupuncture Points for the Treatment of Non-Specific Lower Back Pain. J Altern Complement Integr Med 2: 016

Received: October 15, 2016; Accepted: November 22, 2016; Published: December 07, 2016
[1-2]. Back pain is also one of the most common reasons for missed work, with $80 \%$ of the population experiencing back problems at some time in their lives [2]. According to the CDC, the number of Americans experiencing lower back pain is on the rise, especially amongst over-65 [3].

Acupuncture, a physical intervention which involves placement of small needles in the skin at different acupoints, has been practiced for thousands of years and is commonly used for many types of chronic pain [4-7]. Science has long hypothesized for a scientific explanation of the analgesic successes of acupuncture. Literature supports that acupuncture relieves pain by regulating the autonomic nervous system [8], activating the release of beta-endorphins [9], regulating the central nervous system [10] and producing local effects on the peripheral nervous system [11]. The efficacy of acupuncture for back pain has been supported in literature [12].

Electro-acupuncture has been used as an adjunctive pain management in acupuncture for decades [13] and may be applied invasively or non-invasively. It has been reported to analgesically outperform traditional acupuncture needles [14].

Traditionally, the modality of choice for electro-acupuncture has been Alternating Current (AC) [15-17]. However, there are two known types of electrical currents, AC and Direct Current (DC). DC is uni-directional and is applied microamp or millionth of amp (10-6 amperes) range and is called microcurrent [18-22]. AC moves back and forth and is applied in the miliamperage range (10-3 amperes), and usually called TENS or electro-acupuncture [16]. It is theorized that $\mathrm{AC}$ and DC electro-currents have different modulating affects on the autonomic nervous system and the bodies healing processes $[18,20,22]$.

DC microcurrent therapies involve applying weak direct currents $(80 \mu \mathrm{A}-<1 \mathrm{~mA})$, and are now being increasingly recognized as an adjunct for pain relief and Autonomic Nervous System (ANS) regulation [20-22]. There is no consensus in the literature identifying the best practice measures for application of DC microcurrent to acupuncture points for chronic back pain patients. Although sufficient evidence supports the application of AC-electro-acupuncture and acupuncture needles for back pain, there is no "standardized acupuncture protocol" and limited repetitive evidence in literature to support the use of DC electro-therapies for the treatment of back pain. The purpose of this pilot study was to ascertain the impact of DC microcurrent point stimulation on the pain levels of a cohort of patients immediately post application and 48 hours later after a single MPS application.

\section{Materials and Methods}

The objective data collection was aimed at revealing whether MPS when applied to standard pain protocol can modulate VAS pain scale in patients suffering from back pain. This was an observational study; it is recognized that this analysis was not intended to be one with controls, randomization and inclusions/exclusions criteria nor a comparison with other pain mediating methods like the use of analgesic agents. 


\section{Subjects}

This study entailed the use of MPS in 68 patients (50 female, 18 male; mean age 47 years, SD 1.49) with chronic non-specific neck pain with a mean pain duration of 6.46 years (SD 1.20), presenting to us for therapy of their problem. There were no drop outs within this sample. Inclusion criteria were simple: patients who were currently suffering from chronic neck pain for greater than 3 months, with a recorded VAS pain scale score of $>4$. The diagnoses of pain, location, severity and sex, previous interventions or surgeries were not considered exclusion criteria. Informed consent was obtained to partake in treatment and the study assessments. Patient pain scores were recorded immediate pre treatment and twice post treatment: immediately after application and again two days later.

\section{Methods}

MPS was applied to Standard Protocol using [19] Dolphin Neurostim (Acumed Medical LTD, Ontario, Canada) device. This is an FDA-approved device which apply low frequency, concentrated and microcurrent stimulation (at $10 \mathrm{~K}$ ohms) for the relief of chronic pain [19]. MPS application time was 30 seconds per point, for a total of 16 points located in the lower back, hips and legs (Figure 1). Device was set to negative polarity (-).

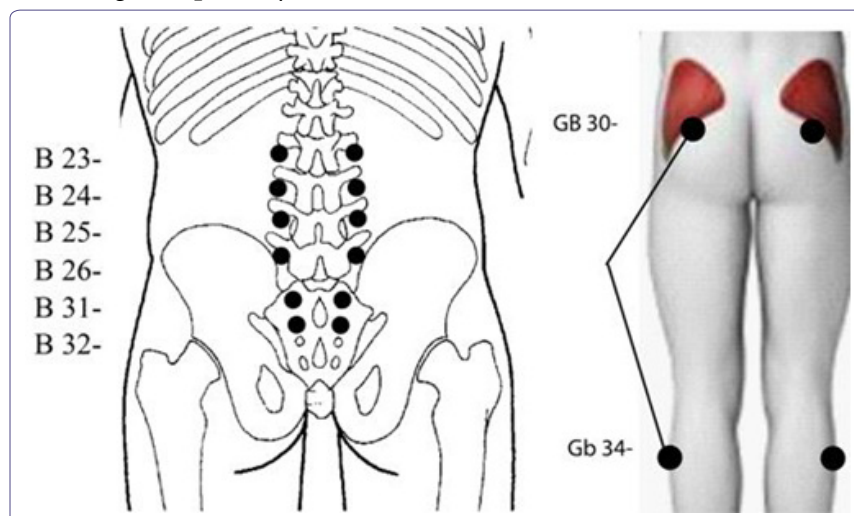

Figure 1: Lower spinal points (left) and acupressure points showing the muscle points and $\mathrm{Gb}$ (Gall bladder meridian) points (right).

Visual Analogue Scale (VAS) was used to evaluate the patient's pain. The VAS is an 11-point scale from 0 - 10 with 0 being no pain and 10 being the most intense pain imaginable [23-25]. The patient verbally selects a value that is most in line with the intensity of the pain that they have experienced in the last 24 hours or is often reported as a rating during a specific movement pattern or functional task. The VAS has good sensitivity [26] and excellent test-retest reliability.

Standard Protocol was developed by BF (Dr Bruce Fashong), as treatment approach to provide a simple, easy to apply, non-pharmaceutical solution for the treatment of chronic pain. The protocol involves the application of concentrated microcurrent stimulation to acupuncture points located in the paraspinal lumbar, hips and legs that isolate the key nerves and muscles of the belly, mid and lower back called the torso or core of the body. This area is highly influential on overall functional movements throughout the rest of the body [27]. When these acupuncture points are collectively treated with concentrated microcurrent, it has been reported that a wide variety of neuromyofascial pain syndromes can be effectively relieved in a timely basis. Standard protocol acupuncture points include the following points (Figure 1).
Statistical analyses were done by 3rd party freelance statistician using paired sample T-test in SPSS software, a widely used program for statistical analysis in social and medical science.

\section{Results}

The VAS response in the cohort of patients with chronic pain following the application of MPS applied to standard acupuncture pain protocol reflected a statistically significant reduction of 3.088 (95\% CI $(2.715,3.462) ; \mathrm{P}=0.001)$. This corresponds to a mean reduction of $62 \%$; from 5.00 to 1.88 , in pain levels post treatment when compared to initial pain levels. There was a further statistically significant reduction of $0.632(33 \%)$ in mean pain levels 48 hours after treatment, when compared to pain levels post treatment $[95 \% \mathrm{CI}(0.295,0.969)$; $\mathrm{p}=0.0001$ ], for a total end pain relief of $75 \%$ (Tables $1 \& 2$; Figure 2).

\begin{tabular}{|c|c|c|c|c|c|c|}
\hline \multicolumn{7}{|c|}{ One-Sample Test } \\
\hline & \multirow{5}{|c|}{$\mathrm{t}$} & $\mathrm{df}$ & $\begin{array}{c}\text { Sig. } \\
(2-\text {-tailed) }\end{array}$ & $\begin{array}{c}\text { Mean } \\
\text { Difference }\end{array}$ & \multicolumn{2}{|c|}{$\begin{array}{c}\text { 95\% Confidence } \\
\text { Interval Of The } \\
\text { Difference }\end{array}$} \\
\cline { 1 - 4 } & & & 0 & 4.985 & 4.52 & 5.45 \\
\hline $\begin{array}{c}\text { Pre Pain } \\
\text { Level (0-10) }\end{array}$ & 21.325 & 67 & 0 & Lower & Upper \\
\hline
\end{tabular}

Table 1: Descriptive statistics of sample T-test with upper and lower values. Upper limit of the box $\sim 7.09$

Lower limit of the box $\sim 3.05$

\begin{tabular}{|c|c|c|c|c|c|}
\hline \multicolumn{5}{|c|}{ Paired Samples Statistics } \\
\hline & Mean & $\mathrm{N}$ & Std. Deviation & \% Pain Reduction \\
\hline & Pre pain level & 5 & 68 & 1.90835 & $0.00 \%$ \\
\hline Post Pain level & 1.8835 & 68 & 1.736 & $-62 \%$ \\
\hline Follow up pain level & 1.2647 & 68 & 1.50241 & $-74.80 \%$ \\
\hline
\end{tabular}

Table 2: Descriptive pain statistics measure of mean, the sample size and a measure of dispersion in standard deviation. This shows the average pain level before therapy is 5.000 (lower variability of 1.90835) compared to the average pain level post application of 1.88 (lower variable 1.736), and follow up pain level of 1.2647 and a variability of 1.50241 . Pain levels reduced from 5.00 to 1.2647 at a confidence levels of $95 \%(p<=0.0001)$.

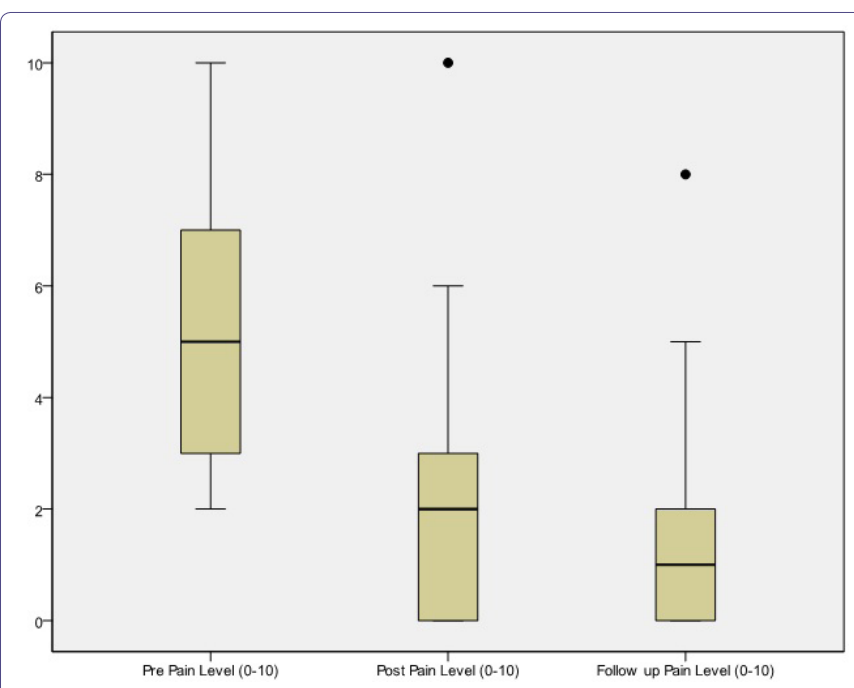

Figure 2: Back pain scores before, immediately post and 48 hours after treatment with MPS applied to low back and gall bladder meridians. 


\section{Discussion}

Chronic Back pain often equates to stress and pain which can make our daily lives miserable, and can lead to significantly impaired physical health [2-3].

The data in this case study clearly shows that the application of MPS to standard protocol provided statistically significantly improved pain outcomes; both immediately post application and two days follow-up for the treatment of non-specific back pain. The consistency of back pain outcomes produced through the treatment of acupuncture points of the standard protocol suggests there is strong relationship between back pain and the muscles and nerves relating to the core (torso?) of the body. It is of interest that the effect of a single application as per protocol continued to show further pain reduction at the 48 hour post evaluation. How long this effect persists needs further study and will help to set guidelines for the frequency of MPS therapy. Since data on analgesic consumption were not available for comparison before and up to 2 days after treatment this analysis cannot predict a possible longer-term use of analgesia and requires further investigation.

It is suggested that low-amplitude DC current mimics human bio-cellular communications, and its application may produce a shift or change in autonomic nervous system resulting in a body wide therapeutic benefits $[20,28]$. These biochemical processes may provide a plausible explanation for the prolonged pain relief after DC microcurrent, and is an area where future research is required. We have already reported, in a single case study, a modification in autonomic nervous system parameters (sympathetic and parasympathetic balance) parallel to a reduction in pain score in a patient with post concussion using MPS stimulation [20]. It is possible that this same mechanism of action is not at play as in this cohort analysis case report, as the pain was not at the same location; this has to be confirmed in other patients.

Furthermore, MPS might have the same effects as EA, provided that the skin impedance allows a sufficient part of the delivered electrical charges to access the subcutaneous structures.

In conclusion, this study showed MPS provided significant (62\%) overall improvements in patient pain levels immediately after initial treatment, and a further significant (30\%) at the two-day follow-up, for a total $75 \%$ pain relief overall improvement when applied to a standard protocol in patients with chronic back pain, suggesting a possible future role for both these modalities in the management of pain or stress related diseases.

Chronic back pain can limit quality of life, restrict work and social engagement, and is often blamed for the development drug dependency of various forms [29]. The changes produced in the autonomic nervous system functions help validate the potential application of MPS to standard protocol as an option to clinicians treating patients with related chronic back pain. However, long term further investigation is warranted with another focus group to confirm these results and to assess their duration.

\section{Acknowledgement}

Statistical analyses were done by 3 rd party freelance statistician using paired sample T-test in SPSS software, a widely used program for statistical analysis in social and medical science.

\section{Author Disclosure Statement}

None of the authors have any commercial associations that create a conflict of interest in connection with submitted manuscript.

\section{References}

1. Jensen MC, Brant-Zawadzki MN, Obuchowski N, Modic MT, Malkasian D, et al. (1994) Magnetic resonance imaging of the lumbar spine in people without back pain. N Engl J Med 331: 69-73.

2. Vällfors B (1985) Acute, subacute and chronic low back pain: clinical symptoms, absenteeism and working environment. Scand J Rehabil Med Suppl 11: 1-98.

3. Center For Disease Control and Prevention (2014) Severe headache or migraine, low back pain, and neck pain among adults aged 18 and over, by selected characteristics: United States, selected years 1997-2013. Center For Disease Control and Prevention, Atlanta, GA, USA

4. Vickers AJ, Cronin AM, Maschino AC, Lewith G, MacPherson H, et al. (2012) Acupuncture for chronic pain: individual patient data meta-analysis. Arch Intern Med 172: 1444-1453.

5. Hinman RS, McCrory P, Pirotta M, Relf I, Forbes A, et al. (2014) Acupuncture for chronic knee pain: a randomized clinical trial. JAMA 312: 1313-1322.

6. Witt C, Brinkhaus B, Jena S, Linde K, Streng A, et al. (2005) Acupuncture in patients with osteoarthritis of the knee: a randomised trial. Lancet 366 : 136-143.

7. Vas J, Perea-Milla E, Méndez C, Sánchez Navarro C, León Rubio JM, et al. (2006) Efficacy and safety of acupuncture for chronic uncomplicated neck pain: a randomised controlled study. Pain 126: 245-255.

8. Li Q-Q, Shi G-X, Xu Q, Wang J, Liu C-Z, et al. (2013) Acupuncture effect and central autonomic regulation. Evid Based Complement Alternat Med 2013: 1-6.

9. Chang RS, Pomeranz B (1979) Electroacupuncture analgesia could be mediated by at least two pain-relieving mechanisms; endorphin and non-endorphin systems. Life Sci 25: 1957-1962.

10. Wu MT, Hsieh JC, Xiong J, Yang CF, Pan HB, et al. (1999) Central nervous pathway for acupuncture stimulation: localization of processing with functional MR imaging of the brain--preliminary experience. Radiology 212: 133-141.

11. Schröder S, Liepert J, Remppis A, Greten JH (2007) Acupuncture treatment improves nerve conduction in peripheral neuropathy. Eur J Neurol 14: 276281.

12. Furlan $A D$, van Tulder $M$, Cherkin $D$, Tsukayama $H$, Lao L, et al. (2005) Acupuncture and dry-needling for low back pain: an updated systematic review within the framework of the cochrane collaboration. Spine (Phila $\mathrm{Pa} 1976$ ) 30: $944-963$

13. Chinese Academy of Traditional Chinese Medicine (1975) An outline of Chinese acupuncture. Foreign Languages Press, Beijing, China.

14. Inoue M, Nakajima M, Hojo T, Kitakoji H, Itoi M (2012) Spinal nerve root electroacupuncture for symptomatic treatment of lumbar spinal canal stenosis unresponsive to standard acupuncture: a prospective case series. Acupunct Med 30:103-108.

15. Robinson AJ, Snyder-Mackler L (2008) Clinical Electrophysiology: Electrotherapy and Electrophysiologic Testing (3rdedn). Lippincott Williams \& Wilkins, New York, USA.

16. Ezzo JM, Richardson MA, Vickers A, Allen C, Dibble SL, et al. (2006) Acupuncture-point stimulation for chemotherapy-induced nausea or vomiting. Cochrane Database Syst Rev.

17. Dang Yi (1999) Acupuncture and Moxibustion. Academy Press, Beijing, China.

18. Armstrong K (2016) Electrotherapy Exposed. Rehab Management, USA 
19. http://www.accessdata.fda.gov/cdrh docs/pdf13/K133789.pdf

20. Chevalier A, Armstrong K, Norwood-Williams C, Gokal R (2016) DC Electroacupuncture Effects on Scars and Sutures of a Patient with Postconcussion Paina. Med Acupunct 28: 223-229.

21. McMakin CR (2004) Microcurrent therapy: a novel treatment method for chronic low back myofascial pain. Journal of Bodywork and Movement Therapies 8:143-153

22. Cheng N, Van Hoof H, Bockx E, Hoogmartens MJ, Mulier JC, et al. (1980) The effects of electric currents on ATP generation, protein synthesis, and membrane transport of rat skin. Clin Orthop Relat Res 264-272.

23. Krebs EE, Carey TS, Weinberger M (2007) Accuracy of the pain numeric rating scale as a screening test in primary care. J Gen Intern Med 22: 14531458.

24. Williamson A, Hoggart B (2005) Pain: a review of three commonly used pain rating scales. J Clin Nurs 14: 798-804.
25. Jensen MP, McFarland CA (1993) Increasing the reliability and validity of pain intensity measurement in chronic pain patients. Pain 55: 195-203.

26. Hawker GA, Mian S, Kendzerska T, French M (2011) Measures of Adult Pain: Visual Analog Scale for Pain (VAS Pain), Numeric Rating Scale for Pain (NRS Pain), McGill Pain Questionnaire (MPQ), Short-Form McGill Pain Questionnaire (SF-MPQ), Chronic Pain Grade Scale (CPGS), Short Form-36 Bodily Pain Scale (SF-36 BPS), and Measure of Intermittent and Constant Osteoarthritis Pain (ICOAP). Arthritis Care Res (Hoboken) 63: 240-252.

27. Karageanes SJ (2005) Principles of manual sports medicine. Lippincott Williams \& Wilkins, New York, USA.

28. Uy J, Ridding MC (2003) Increased cortical excitability induced by transcranial DC and peripheral nerve stimulation. J Neurosci Methods 127: 193-197.

29. Maniadakis N, Gray A (2000) The economic burden of back pain in the UK Pain 84: 95-103. 